Instrumental Background

\section{Opening a Window on the Universe}

Charles Mollan

It was Noah and his family who saw the first rainbow (or so the bible tells us). But it was a while before scientists got in on the act of discovery. Isaac Newton (16421727 ), in 1666, procured "a triangular glass-Prisme, to try therewith the celebrated Phaenomena of Colours". By splitting white light into its colours using his prism, and recombining them, he found "that light itself is a Heterogeneous mixture of differently refrangible rays". $\mathrm{He}$ thus explained the rainbow, in which rain drops act as prisms.

But it appears to have been the co-incidence of two exceptionally able scientists, in the same place at the same time, which led to another revolutionary scientific discovery. One was a chemist, Robert Bunsen (1811-1899), well known for the introduction of a rather unsophisticated piece of apparatus which nevertheless has had a profound effect on laboratory science, the ubiquitous Bunsen burner. The other was a physicist, Gustav Kirchhoff (1824-1887). And both were professors at Heidelberg. Kirchhoff was investigating the dark lines which Josef von Fraunhofer (1787-1826) had observed in the spectrum of the Sun, and by 1859 he reckoned that they were caused by absorption of certain wavelengths by substances in the Sun's atmosphere. At the same time Bunsen was looking at the colours given off by heating chemicals to incandescence. Kirchhoff suggested that by looking at the spectral lines seen when Bunsen's colours were passed through a prism, they would have a precise way of checking the colour. What they found was that each substance had its own unique spectrum. By 1861 they had used their new method of spectroscopic analysis to find two new elements, caesium and rubidium.

The method was almost immediately seen to be of dramatic importance to

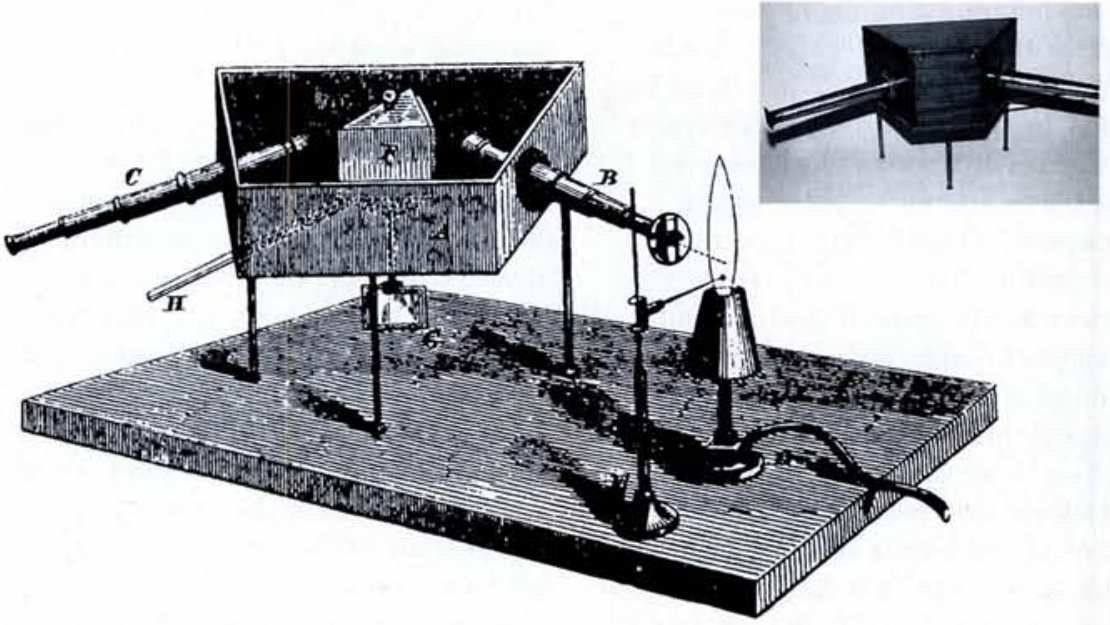

Fig 1 The primitive spectroscope used at first by Kirchhoff and Bunsen.

The substance to be investigated is inserted in the flame and a 'collimator' with a thin slit at its right hand end carries an image of the flame to the prism, which is contained in a darkened chamber. The prism separates

astronomy, and a contemporary commentator (Richard Proctor, 1869) soon wrote, "but now a new and wonderful mode of inquiry has been devised, and has rapidly taken its place as the most important of all the methods of research which science has as yet placed in the hands of her servants. I refer to spectroscopic analysis, or the analysis of light by means of the prism. This mode of research is one to which the powers of great telescopes are admirably adapted.... The whole power of the new method of research depends on the emission of light from an object. It matters not whether the object be in the laboratory of the chemist, or half a mile off, or a hundred millions of miles off, or in fine as far off as the most distant star; if we can only obtain light enough from it to form a distinct spectrum, we can tell what is its nature."

Since Kirchhoff and Bunsen's discovery in 1860 , spectroscopes have evolved dramatically (see figures), extending from the visible in both directions to other bands of

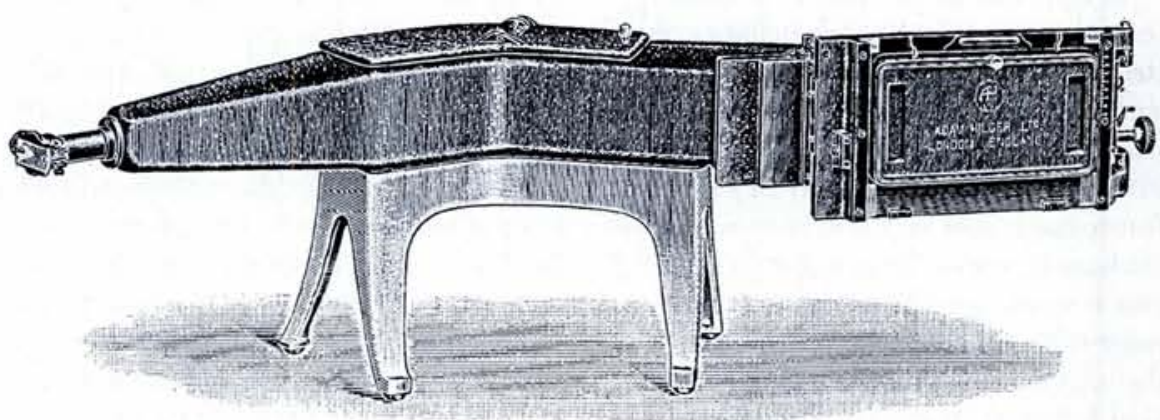

Fig 3 A quartz photographic spectrograph by Adam Hilger, London, 1931, for use from $2000 \AA$ in the ultra- the colours, and the resulting spectrum is observed through the left hand telescope

Fig 2 Photograph A contemporary copy of the first spectroscope (unsigned), circa 1860, preserved in the collection of University College physics department at Galway, Ireland

the electromagnetic spectrum. Science has become ever more colourful since those pioneering days in Germany in the late 1850 s and early 1860 s. Our knowledge of the universe and of our own world has increased enormously as a result. Many physics departments have a range of old spectroscopes for teaching and research. Because of their importance they are well worth preserving and displaying, particularly as their descendants are still playing vital roles in the making of great discoveries. One such recent discovery was the football-shaped 60-carbon-atom molecule buckminsterfullerene. Work on the fullerenes led to the award of the 1996 Chemistry Nobel Prize to Robert Curl, Harold Kroto and Richard Smalley. Sadly, however, modern spectroscopes are not so elegant as were their ancestors.

Further Reading: James Bennett, The Celebrated Phaenomena of Colours, Whipple Museum, Cambridge, 1984. 